\title{
Double Knockdown of PHD1 and Keap1 Attenuated Hypoxia-Induced Injuries in Hepatocytes
}

\author{
Jing Liu, Yiping Li, Lei Liu, Zhi Wang, Chuanbing Shi, Zhengyuan Cheng, Xiaoyi Zhang, \\ Fengan Ding and Ping Sheng Chen* \\ Department of Pathology and Pathophysiology, School of Medicine, Southeast University, Nanjing, China
}

Background and Aims: Hypoxia and oxidative stress contribute toward liver fibrosis. In this experiment, we used small hairpin RNA (shRNA) to interfere with the intracellular oxygen sensor-prolyl hydroxylase 1 (PHD1) and the intracellular oxidative stress sensor-kelch-like ECH associated protein 1 (Keap1) in the hypoxic hepatocytes in order to investigate the function of PHD1 and Keap1.

Methods: We first established the $\mathrm{CCl}_{4}$-induced liver fibrosis model, subsequently, the levels of the PHD1, hypoxia-inducible factor- $1 \alpha(\mathrm{HIF}-1 \alpha)$, hypoxia-inducible factor-2 $\alpha$ (HIF-2 $\alpha$ ), Keap1, and nuclear factor-erythroid 2 p45-related factor 2 (Nrf2) were detected in liver tissues. Simultaneously, AML12 cells co-transfected with PHD1

OPEN ACCESS

Edited by:

Maria Giovanna Trivella, Consiglio Nazionale Delle Ricerche (CNR), Italy

Reviewed by:

Katja Breitkopf-Heinlein, Heidelberg University, Germany Antonella Cecchettini,

University of Pisa, Italy

*Correspondence:

Ping Sheng Chen

101006524bingli@sina.cn

Specialty section: This article was submitted to Integrative Physiology, a section of the journal Frontiers in Physiology

Received: 22 October 2016 Accepted: 21 April 2017 Published: 10 May 2017

Citation: Liu J, Li Y, Liu L, Wang Z, Shi C, Cheng $Z$, Zhang $X$, Ding $F$ and Chen PS (2017) Double Knockdown of PHD1 and Keap1 Attenuated

Hypoxia-Induced Injuries in Hepatocytes. Front. Physiol. 8:291.

doi: 10.3389/fphys.2017.00291 and Keap1shRNAs were constructed in vitro, then the intracellular oxidative stress, the proportion of cells undergoing apoptosis, and cell viability were measured. The expression of pro-fibrogenic molecules were analyzed via quantitative real-time polymerase chain reaction (qRT-PCR) and western blot. The level of alpha- 1 type I collagen (COL1A1) was determined using an enzyme-linked immunosorbent assay (ELISA). Finally, serum-free "conditioned medium" (CM) from the supernatant of hypoxic AML12 hepatocytes was used to culture rat hepatic stellate cells (HSC-T6), and the levels of fibrosis-related molecules, apoptosis, and cell proliferation were determined.

Results: The marker of hypoxia-HIF-1 $\alpha$ and HIF-2 $\alpha$ in the livers with fibrosis were upregulated, however, the increase in PHD1 expression was not statistically significant in comparison to the control group. Sign of oxidative stress-Keap1 was increased, while the expression of Nrf2, one of the Keap1 main downstream molecules, was reduced in the hepatocytes. And in vitro, the double-knockdown of PHD1 and Keap1 in AML12 hepatocytes presented with decreased hypoxia-induced oxidative stress and apoptosis, furthermore, these hypoxic AML12 cells showed the increased cell viability and the doweregulated expression of pro-fibrogenic molecules. In addition, HSC-T6 cells cultured in the hypoxic double-knockdown CM demonstrated the downregulation of fibrosis-related molecules, diminished cell proliferation, and enhanced apoptosis.

Conclusions: Our study demonstrated that double-knockdown of PHD1 and Keap1 attenuated hypoxia and oxidative stress induced injury in the hepatocytes, and subsequently inhibited HSC activation, which offers a novel therapeutic strategy in the prophylaxis and treatment of liver fibrosis.

Keywords: liver fibrosis, hepatocytes, PHD1, Keap1, hypoxia, oxidative stress 


\section{INTRODUCTION}

Liver fibrosis is a pathological process caused by numerous chronic liver damages-such as viral hepatitis, alchoholic hepatitis, and drug induced hepatotoxicity (Begriche et al., 2011). As a highly active metabolic organ, the liver is particularly prone to hypoxic environments and the damages caused by it (Nakanishi et al., 1995). A convincing body of evidence suggest that hypoxia plays an important role in the pathogenesis of liver fibrosis (Cannito et al., 2014).

Cellular hypoxia leads to activation of hypoxia-inducible factors (HIF), which is crucial for the survival of an asphyxiated/ischemic hepatocyte. In normoxia, HIF-1 $\alpha$ and HIF-2 $\alpha$ are first hydroxylated by prolyl hydroxylase (PHD) and then degraded by proteasomes (Kamura et al., 2000). Whereas during hypoxic condition, HIF- $1 \alpha$ and HIF- $2 \alpha$ accumulate and get translocated to the nuclei, thereby activating the genes responsible for limiting hypoxia-induced injury and cell death. Recently, Nimker et al. reported that the pharmaceutical inhibition of PHDs with ethyl 3, 4-dihydroxy benzoate (EDHB) protects myoblasts against hypoxia-induced oxidative damage by upregulating HIFs (Nimker et al., 2015). However, non-specific inhibition of PHDs also induces the activation of HIFs but at the cost of adverse effects such as steatosis (Minamishima et al., 2009; Rankin et al., 2009). PHD has three isoforms-PHD1, PHD2, and PHD3, each of these has numerous functions. Selective loss of PHD1, but not those of PHD2 or PHD3 could induce hypoxia tolerance in the skeletal muscle and liver cells via reprogramming of basal oxygen metabolism without inducing angiogenesis and erythrocytosis (Aragones et al., 2008; Schneider et al., 2010). Therefore, the specific inhibition of PHD1 could serve as a potential therapeutic strategy against hypoxia during liver fibrosis.

In recent years, many studies have demonstrated that oxidative stress also contributes to the pathogenesis of liver fibrosis (Ghatak et al., 2011; Mormone et al., 2012; Yang et al., 2013).The nuclear factor-erythroid 2-related factor 2 (Nrf2) has been suggested to be involved in this process. Under normal condition, Nrf2 exists in the cytoplasm where it binds to kelchlike ECH associated protein 1 (Keap1), thereby hastening the Nrf2 ubiquitination and degradation (Kang et al., 2004). During oxidative stress, Nrf2 evades Keap1 and translocates to the nucleus, where it activates antioxidant genes. Chen et al. has also demonstrated that glycyrrhetinic acid could ameliorate chronic liver fibrosis via upregulation of Nrf2 (Chen et al., 2013). Additonally, Tanaka et al. also reported that Nrf2-null mice had an increased susceptibility to liver injury (Tanaka et al., 2012). Keap1 serves to negatively regulate Nrf2 (Miyata et al., 2011), for example, Keap1-knockdown mice are less vulnerable to oxidative liver injuries during obstructive cholestasis mediated by enhanced expression of Nrf2 (Okada et al., 2009). Thus, Keap1 may be an efficient therapeutic target for relieving oxidative stress injury during liver fibrosis.

It has been reported that many cell types are involved in the pathogenesis of liver fibrosis, activated hepatic stellate cells (HSCs) play a central role in liver fibrogenesis. It is worth noting that injuried hepatocytes are considered to be the primary activator of HSCs (Nieto et al., 2002), and hepatocytes are the major parenchymal cells that account for more than $70 \%$ of all liver cells (Bogdanos et al., 2013). Furthermore, hepatocytes are highly susceptible to hypoxic injury and the drugs induced toxicity, therefore we chose hepatocytes as a therapeutic target in an attempt to ameliorate liver fibrosis. Hypoxic hepatocytes are known to generate excessive amount of reactive oxygen species (ROS) which exert oxidative stress on the liver. Moreover, such oxidative stress can further exacerbate the hypoxia in the hepatocytes, and the subsequent re-oxygenation following hypoxia leads to additional oxidative stress (Miyata et al., 2011). Therefore, the ideal therapy for liver fibrosis could be the alleviation of hypoxia along with the oxidative stress, however, no such related studies have been done so far.

RNA interference (RNAi) is a method that uses a small complementary double-stranded RNA (dsRNA) molecule to silence a target gene (Inoue et al., 2006). Considering the short half-life and high cost of current small interfering RNA (siRNA) vectors, they are not suitable for many in vivo studies. In contrast, short hairpin RNA (shRNA) is widely used due to its enhanced stability and transfer efficiency. Accordingly, for the present study we used shRNAs to simultaneously knockdown the expressions of PHD1 and Keap1 in the hepatocytes with the aim of exploring therapeutic target for liver fibrosis.

\section{MATERIALS AND METHODS}

\section{Animals and Treatment}

Six-week-old male Sprague-Dawley rats $(250 \pm 30 \mathrm{~g})$ were purchased from the Animal Center of Yangzhou University (Yangzhou, China). All animals were housed in the animal experimental center of Southeast University under constant temperature $\left(22^{\circ} \mathrm{C}\right)$ and humidity $(55 \pm 5 \%)$ in a controlled room with a $12-12 \mathrm{~h}$ light-dark cycle, where the diet and water were available ad libitum. The rats were acclimatized under these conditions for at least 1 week prior to experiments. All animals were randomly divided into two groups: a treatment group $(n=10)$ which received $1.5 \mathrm{ml} / \mathrm{kg}$ body weight carbon tetrachloride $\left(\mathrm{CCl}_{4} ; 40 \% \mathrm{CCl}_{4}\right.$ in olive oil) via intraperitoneal injection twice a week for eight weeks and a normal control group $(n=10)$ which received a saline injection in parallel. This study was carried out in accordance with the recommendations of the European Council Directive of the 24th November 1986 (86/609/EEC). All procedures were approved by the Animal Research Ethics Committee at the Medical School of the Southeast University (Nanjing, China).

\section{Histology and Immunohistochemistry}

Excised livers were fixed in $10 \%$ neutral-buffered formalin, embedded in paraffin, and cut into sections of $5 \mu \mathrm{m}$ thickness. Slides were then deparaffinized with dimethylbenzene, dehydrated with graded ethanol, and stained with hematoxylin and eosin (H\&E) and Masson dyes to evaluate the degree of liver fibrosis. 
For immunohistochemical probing, liver slides were initially boiled in a pressure cooker containing a citric acid buffer $(\mathrm{pH}$ $6.0)$ to retrieve antigens. Then the slides were blocked with $5 \%$ BSA and incubated with primary antibodies (Table 1) overnight at $4^{\circ} \mathrm{C}$, and with a goat anti-rabbit biotinylated secondary antibody for $20 \mathrm{~min}$ at $37^{\circ} \mathrm{C}$. Immunolabels were detected with 3, 3-Diaminobenzidine (DAB), after which the nuclei were counterstained with hematoxylin. Slides were inspected under a fluorescence microscope at $200 \times$ magnification. For quantification analysis, the percentage of positive area for immunohistochemistry was determined using ImageJ software.

\section{Cell Cultures and Hypoxia Treatment}

AML12 (alpha mouse liver 12 cells) and HSC-T6 (rat hepatic stellate cells) were purchased from the Shanghai Institute of Biochemistry and Cell Biology. Cells were cultured in

\begin{tabular}{lll}
\hline \multicolumn{2}{l}{ TABLE 1 | The antibody for immunohistochemistry and western blot. } \\
\hline Antibody & Manufacturer & Cat. No. \\
\hline HIF-1 $\alpha$ & Abcam & ab179483 \\
HIF-2 $\alpha$ & Abcam & ab179825 \\
PHD1 & Abcam & ab108980 \\
Nrf2 & Abcam & ab137550 \\
Keap1 & Proteintech & $10503-2-A P$ \\
$\alpha-S M A$ & Proteintech & $14395-1-A P$ \\
COL1A1 & Bioworld & BS60771-25 \\
TGF- $\beta 1$ & Bioworld & BS1361 \\
VEGF-A & Proteintech & $19003-1-A P$ \\
IGF-1 & Bioworld & BS2909 \\
GAPDH & Bioworld & AP0063
\end{tabular}

DMEM-F12 (Hyclone, USA) supplemented with 10\% heatinactivated fetal bovine serum (Bioind, Israel), and were maintained in a humidified atmosphere with $5 \% \mathrm{CO}_{2}$ at $37^{\circ} \mathrm{C}$. For hypoxic exposure, AML12 cells were incubated in a trigas incubator (Thermo, America) of $1 \% \mathrm{O}_{2}, 5 \% \mathrm{CO}_{2}$, and $94 \%$ $\mathrm{N}_{2}$. Serum-free "conditioned medium" (CM) was obtained from the supernatant of AML12 cells to eliminate the influence of serum cytokines. HSC-T6 cells were cultured in CM and the cells cultured in DMEM were used as control.

\section{RNA Isolation and Quantitative Real-Time Polymerase Chain Reaction}

Total RNA was extracted from cells with Trizol reagent (TaKaRa, Japan). Then, $1 \mu \mathrm{g}$ RNA was added to a $20 \mu \mathrm{l}$ reaction volume for cDNA reverse transcription using the Prime Script ${ }^{\mathrm{TM}}$ RT reagent Kit with gDNA Eraser (TaKaRa, Japan), and quantitative real-time polymerase chain reaction (qRT-PCR) was performed using the BR ${ }^{\circledR}$ Premix Ex Taq ${ }^{\mathrm{TM}}$ (TaKaRa, Japan) in a Step One Plus real-time PCR system (Applied Biosystems, USA). Gene expression was quantified according to the $2^{-\Delta \Delta \mathrm{Ct}}$ method. All PCR primers are listed in Table 2.

\section{Protein Extraction and Western Blot}

Cells were lysed with RIPA buffer (Beyotime, China) containing a Protease Inhibitor Cocktail (Roche, Germany) and then centrifuged at $12,000 \times \mathrm{g}$ for $15 \mathrm{~min}$ at $4^{\circ} \mathrm{C}$. Equal amounts of protein were separated via gel electrophoresis and transferred to a PVDF membrane (Millipore Corp, USA). Membranes were blocked with $5 \%$ skimmed milk for $1 \mathrm{~h}$ at $37^{\circ} \mathrm{C}$ and incubated with primary antibodies (Table 1) overnight at $4^{\circ} \mathrm{C}$ and then with corresponding secondary antibodies for $1 \mathrm{~h}$ at $37^{\circ} \mathrm{C}$. The blotting signal was visualized using enhanced chemiluminescence reagent (HaiGene, China). Final protein levels were normalized to that of GAPDH.

TABLE 2 | Primer sequences for quantitative PCR.

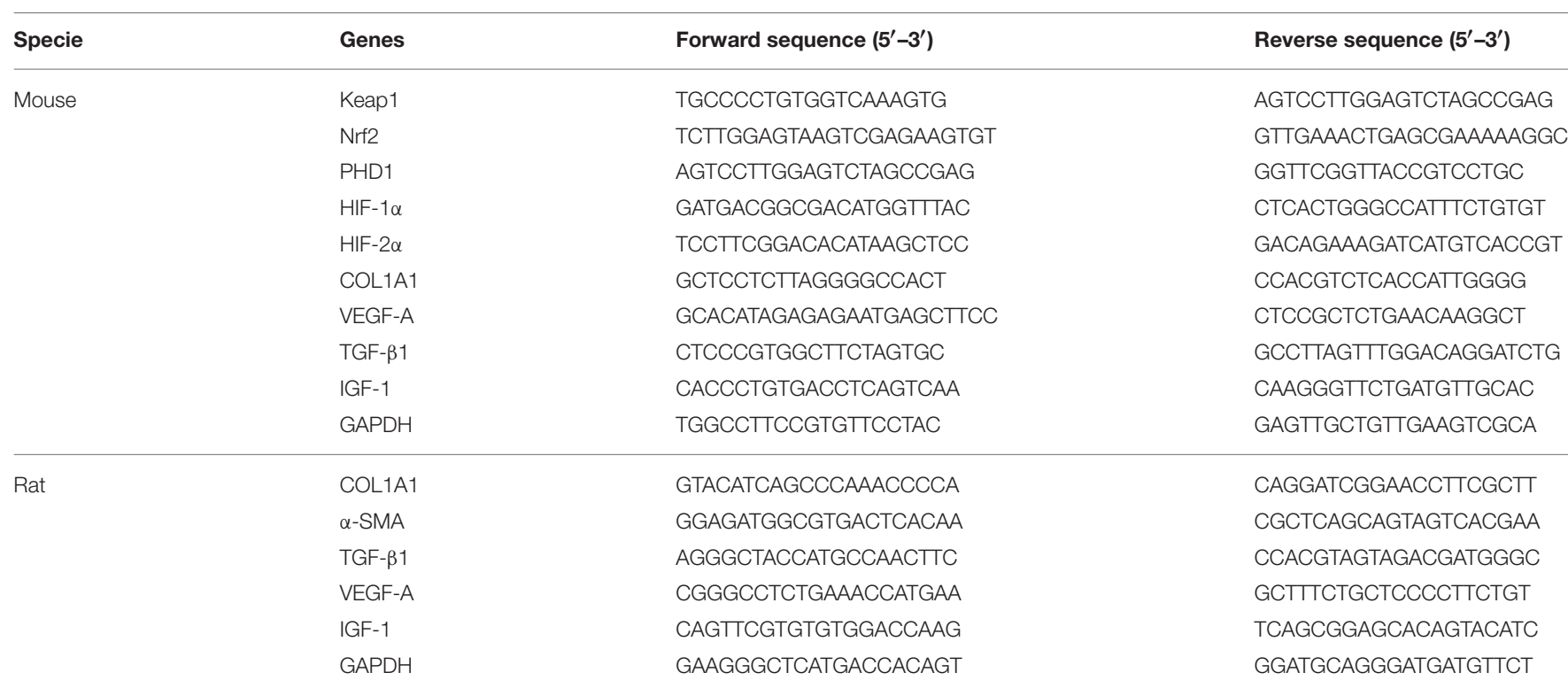




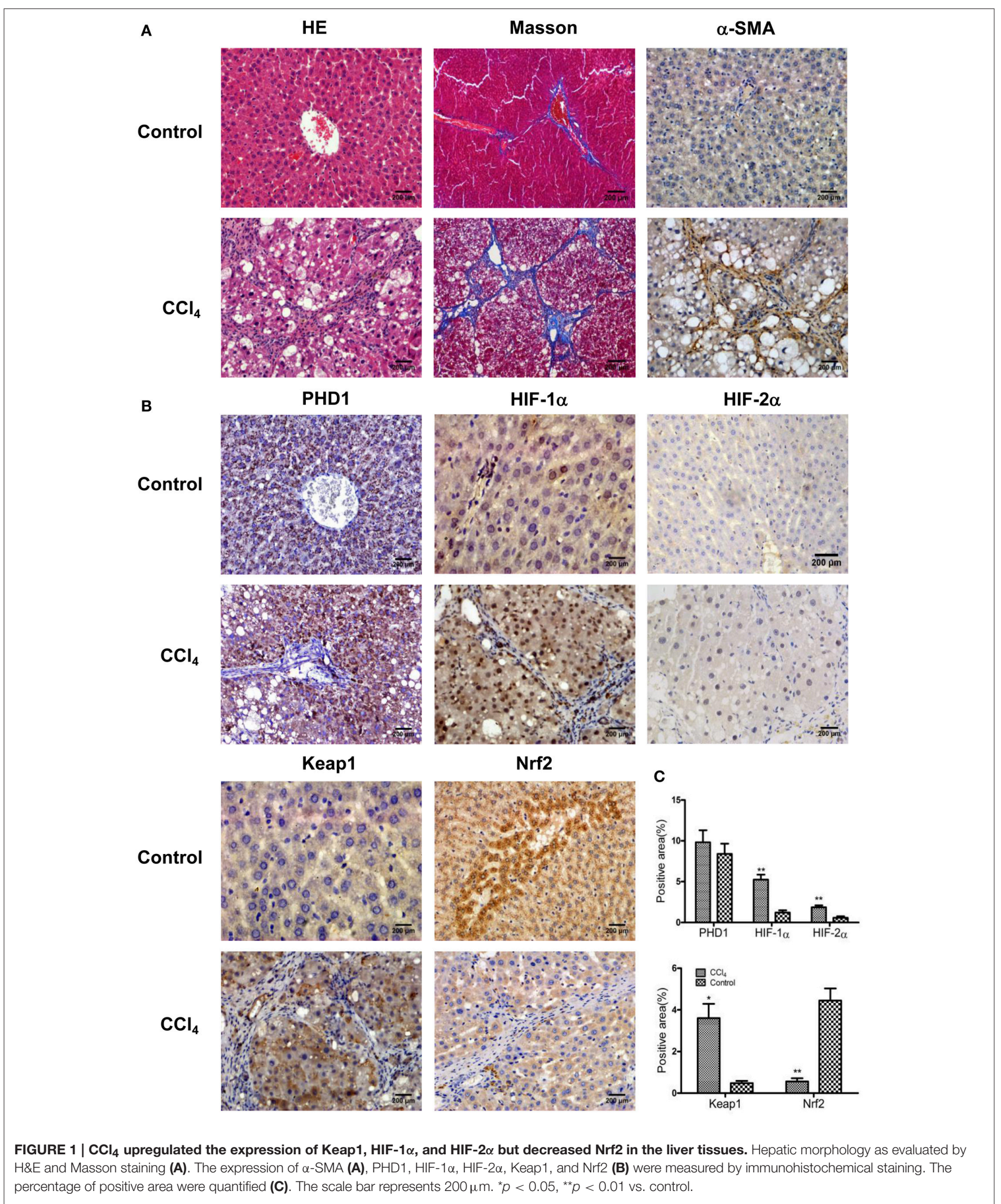



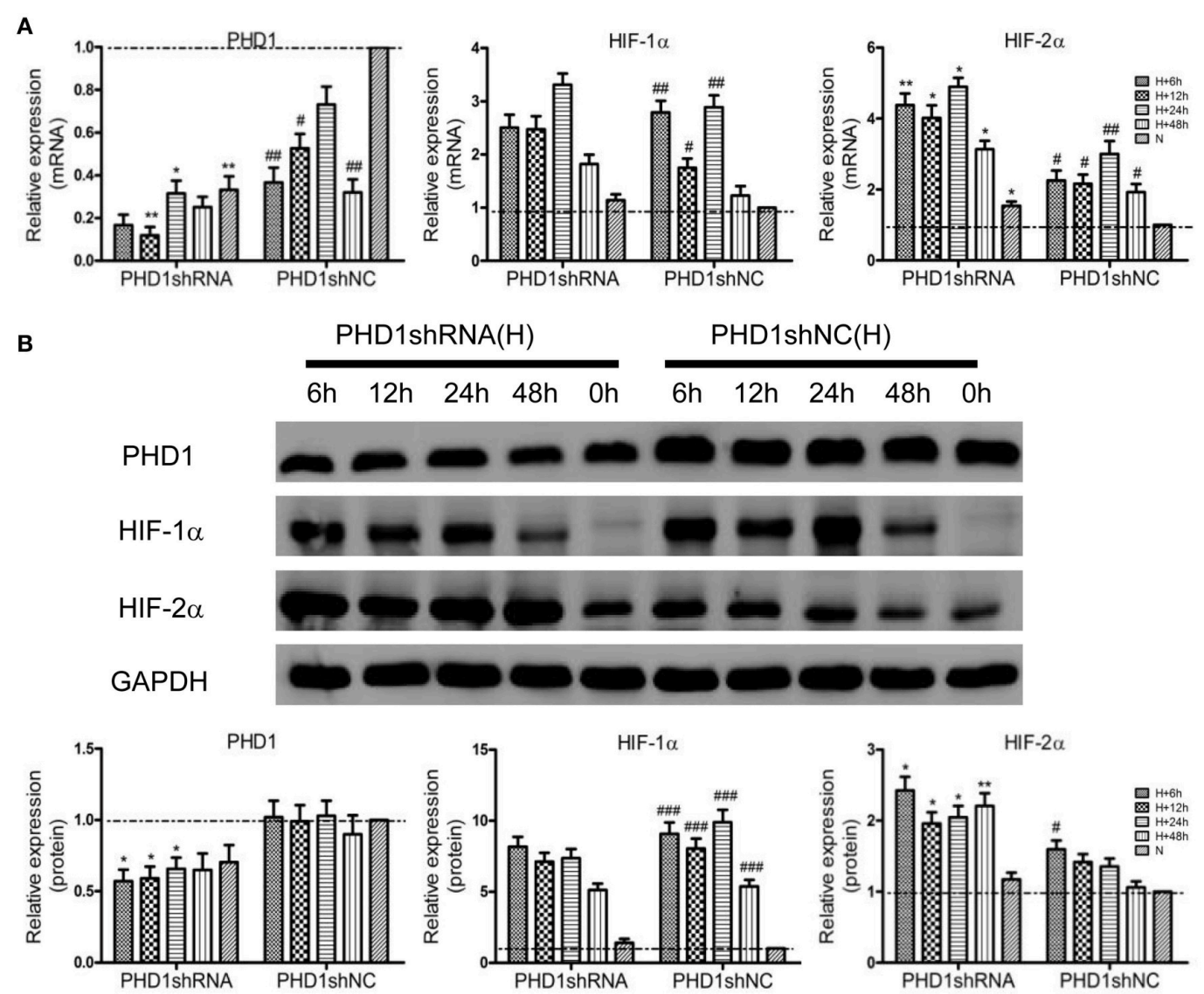

FIGURE 2 | PHD1shRNA upregulated the expression of HIFs in hypoxic AML12 cells at various time interval between 0-48 $\mathrm{h}$. (A) mRNAs and (B) proteins levels of PHD1, HIF-1 $\alpha$ and HIF-2 $\alpha$ were measured by qRT-PCR and western blot, and GAPDH was used as the internal standard, $n=3$. ${ }^{*} p<0.05$, ${ }^{* *} p<0.01$ vs. shNC at equivalent time interval, $\# p<0.05, \# \# p<0.01$, \#\#\# $p<0.001$ vs. shNC under normoxia. N, normoxia; H, hypoxia.

\section{Malondialdehyde (MDA), Reduced Glutathione (GSH) Levels, and Lactate Dehydrogenase (LDH) Activity Assay}

MDA and GSH levels were detected in total cell lysates via commercial assay kits (Jian Cheng Bioengineering Institute, China). All levels are expressed as $\mu \mathrm{mol} / \mathrm{g}$ protein. For detection of LDH activity, supernatants were collected and analyzed with the LDH assay kit (Jian Cheng Bioengineering Institute, China). $\mathrm{LDH}$ activity is expressed as U/L.

\section{Enzyme-Linked Immunosorbent Assay for Collagen Secretion}

The level of alpha-1 type I collagen (COL1A1) in the supernatants of AML12 cells was determined using a commercial enzymelinked immunosorbent assay (ELISA) kit (Hengyuan Biology, China) according to the manufacturer's protocol. The optical density was measured at a wavelength of $450 \mathrm{~nm}$.

\section{Cell Viability Assay}

Cellular viability was measured using the cell counting kit-8 (CCK-8) assay (Dojindo, Japan) following the manufacturer's protocol. Absorbance (OD value) was measured at $450 \mathrm{~nm}$ using an enzyme-linked immunosorbent assay reader (Thermo Fisher Scientific).

\section{Apoptosis Assay}

Cells undergoing apoptosis were stained with the Annexin-V/PI stain (Ebscience, America). For cells that were transfected with plasmids with green and red fluorescence, PI was replaced by DAPI to label apoptosis. A Flow cytometer (Becton Dickinson, USA) was used to quantify apoptotic cells.

\section{Statistical Analysis}

Results are expressed as mean \pm SEM. One-way ANOVA and the Student's $t$-test were used to evaluate any variations in outcomes between different groups. A $p$-value $<0.05$ was considered as statistically significant.

\section{RESULTS}

\section{Hypoxia and Oxidative Stress in the Rats with Liver Fibrosis}

To understand the microenvironment in the fibrotic liver tissues, we established $\mathrm{CCl}_{4}$-induced liver fibrosis rat model. $\mathrm{H} \& \mathrm{E}$ staining showed that $\mathrm{CCl}_{4}$ exposure resulted in severe damage 
of the liver cells, including hepatocytes necrosis, inflammatory cells infiltration, steatosis, and fibrosis connective tissues proliferation. However, the control group presented normal histological morphology (Figure 1A). Masson's trichrome staining imparts a blue color to collagen against the red background of other structures. According to the microscopic examination, $\mathrm{CCl}_{4}$ treatment resulted in an increased collagen deposition around the portal area and formation of septal fibrosis (Figure 1A). Furthermore, immunohistochemical staining demonstrated an increased expression of $\alpha$-smooth muscle actin ( $\alpha$-SMA, a marker of HSC activation, which plays a critical role in liver fibrogenesis) in fibrous septae (Figure 1A). These results indicated that the $\mathrm{CCl}_{4}$-induced liver fibrosis was conducted successfully. Sitmultaneously, we found the upregulation of HIF- $1 \alpha$ and HIF- $2 \alpha$ (markers of hypoxia), and Keap1 (an intracellular oxidative stress sensor) in hepatocytes in response to $\mathrm{CCl}_{4}$ treatment (Figures 1B,C). However, Nrf2, one of the main downstream molecules of Keap1, was downregulated, while PHD1 showed no obvious change (Figures 1B,C).

\section{Effects of PHD1shRNA on HIF Levels in the Hypoxic AML12 Cells}

To clarify the function of PHD1 during hypoxia-induced liver injuries, PHD1shRNA was applied to transfect the AML12 cells, then the cells were exposed to hypoxia for $0,6,12,24$, and $48 \mathrm{~h}$, the expression of PHD1, HIF- $1 \alpha$ and HIF- $2 \alpha$ mRNAs and proteins were measured. As shown in Figure 2A, PHD1mRNA expression was reduced in hypoxic shNC (negative control) cells compared to that in normoxic shNC cells. Introduction of PHD1shRNA inhibited $60-80 \%$ of PHD1 mRNA expression, achieving optimum gene silencing at $12 \mathrm{~h}$ of hypoxia. However, PHD1 protein level was invariably reduced by $~ 50 \%$ regardless of the duration of hypoxia (Figure 2B). Under normoxic condition, the expression of HIF- $1 \alpha$ and HIF- $2 \alpha$ mRNAs (Figure 2A) and proteins (Figure 2B) were in low expression, and upon exposed to hypoxia, these expression were upregulated. Furthermore, PHD1 silencing further increased the expression of HIF- $2 \alpha$ but not that of HIF- $1 \alpha$ at both the mRNA and protein levels.

\section{Effects of Keap1shRNA on Nrf2 Level in the Hypoxic AML12 Cells}

To clarify the effect of Keap1shRNA, the AML12 cells were transfected with Keap1shRNA and were administrated with hypoxia for $0,6,12,24$, and $48 \mathrm{~h}$, then the expression of Keap1 and Nrf2 at the mRNA and protein levels was determined. An $84 \%$ reduction in Keap 1 mRNA (Figure 3A) and 50\% reduction in Keap1 protein (Figure 3B) levels were observed at $12 \mathrm{~h}$ of hypoxia in the cells silenced with Keap1shRNA. As expected, Nrf2 mRNA and protein levels were increased in the hypoxic shNC cells compared to that in the normoxic shNC cells, and the expression of Nrf2 mRNA and protein were further increased in hypoxic cells treated with Keap1shRNA, which peaked at $12 \mathrm{~h}$ of hypoxia (Figures 3A,B).

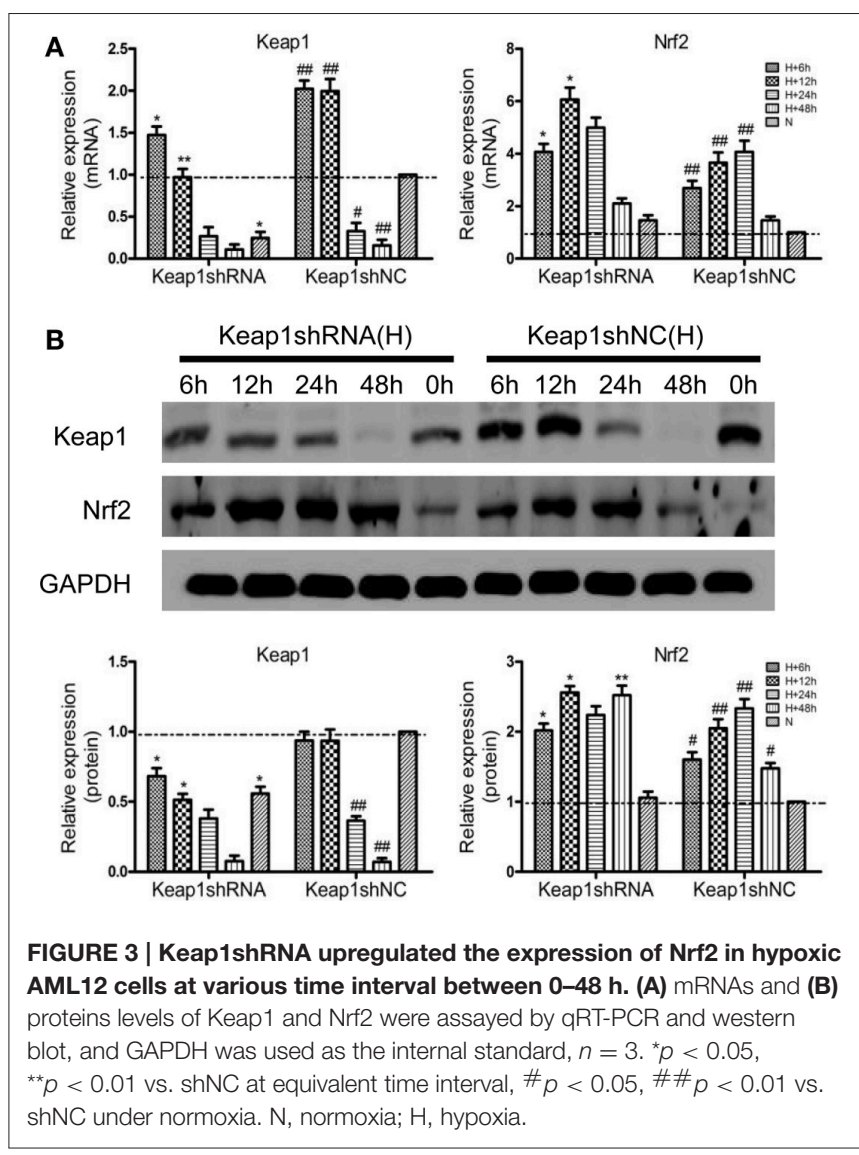

\section{Effects of PHD1 and Keap1shRNAs on Oxidative Stress in the Hypoxic AML12 Cells}

Since hypoxia led to an increase in oxidative stress, we next studied how molecular markers of oxidative stress-MDA and GSH (important lipid peroxidation and endogenous antioxidant marker, respectively) were regulated. The AML12 hepatocytes were co-transfected with PHD1 and Keap1shRNAs, and then were cultured for $12 \mathrm{~h}$ under hypoxia. When cells exposed to hypoxia, a dramatic enhancement of MDA was observed, but the treatment of single knockdown of PHD1 or Keap1 suppressed the increase, furthermore, the double knockdown group was more effective in reducing the hypoxia-induced MDA elevation (Figure 4A). However, no significant differences in the levels of MDA and GSH between gene knockdown cells and control cells during normoxia (Figure 4A), The intracellular GSH concentration presented the inverse correlation with the MDA level (Figure 4A).

\section{Effects of PHD1 and Keap1shRNAs on AML12 Cells Viability during Hypoxia}

To understand the effects of PHD1 and Keap1 molecules on cell biological behavior, the Annexin V-APC/DAPI double staining was used to analyze cell apoptosis. The results were presented as the sum of the percentage of early apoptotic 
A
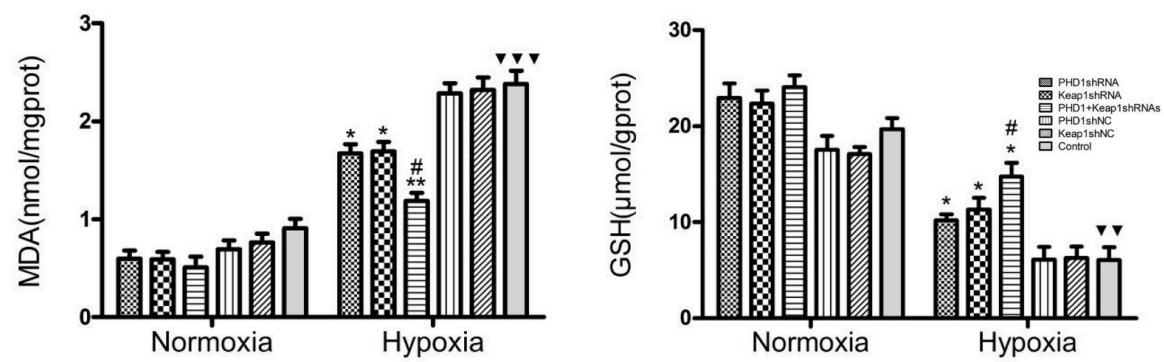

B

PHD1shRNA Keap1shRNA PHD1+Keap1shRNAs PHD1shNC Keap1shNC
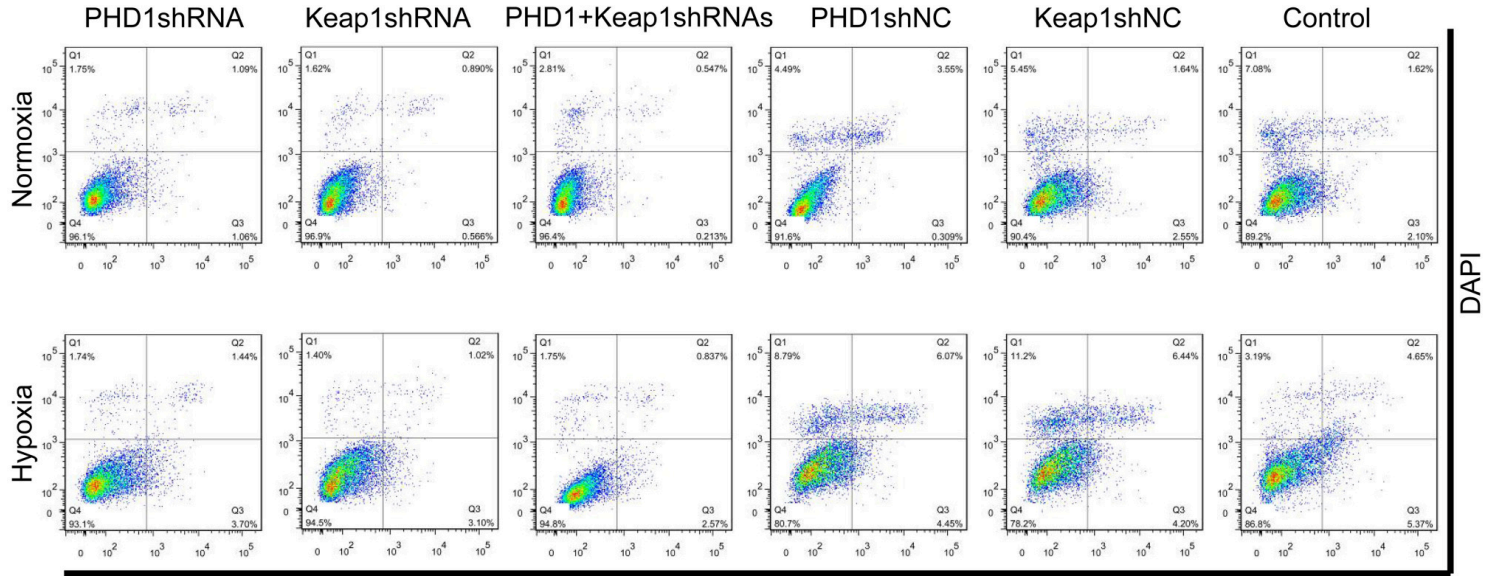

Annexin V APC

C

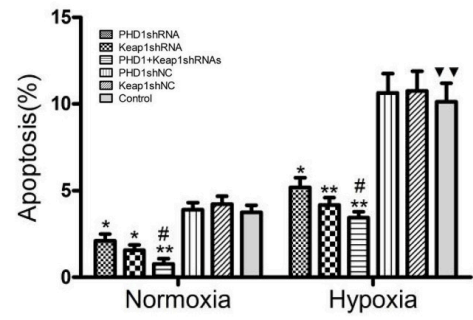

D

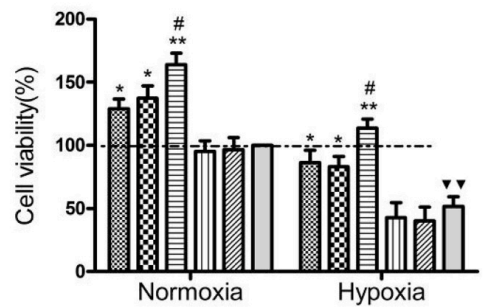

E

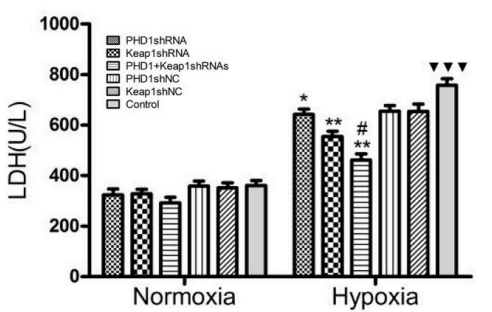

FIGURE 4 | Co-transfection of PHD1 and Keap1shRNAs inhibited oxidative stress and increased cell viability during hypoxia. Co-transfected AML12 cells were exposed to hypoxia for $12 \mathrm{~h}$, and (A) intracellular MDA and GSH levels, (B,C) Annexin V-APC/DAPI double-stained cells undergoing apoptosis, (D) cell viability and (E) LDH levels were assessed. Data are expressed as mean $\pm \mathrm{SEM}, n=3$. ${ }^{*} p<0.05$, ${ }^{\star *} p<0.01$ vs. respective normoxic and hypoxic control, ${ }^{\#} p<0.05$ vs. respective single-knockdown normoxic and hypoxic control, $\mathbf{\nabla} \mathbf{v}_{p}<0.01, \mathbf{\nabla} \mathbf{\nabla} \mathbf{v}_{p}<0.001$ vs. normoxic control.

cells and late apoptotic cells. In Figures 4B,C, during hypoxia, the percentage of apoptotic cells in the single PHD1shRNA $(6.49 \pm 0.50 \%)$ or KeaplshRNA $(5.86 \pm 0.45 \%)$ treatment group were notably lower than in the hypoxic control group $(10.14 \pm 0.79 \%)$. Moreover, the apoptotic rate was further reduced in the double-knockdown group (4.63 $\pm 0.36 \%)$. A similar trend among treatment and control groups were also observed during normoxia.

Next, the cell viability was examined using the CCK- 8 assay. The data in Figure 4D indicated that hypoxia evidently reduced cell viability, however, co-treatment with PHD1 and Keap1 shRNAs led to an increase in cell viability compared to single shRNA transfection. Cellular membrane integrity was analyzed by detecting $\mathrm{LDH}$ release. As shown in Figure 4E, LDH release in different hypoxia groups fluctuated with a similar tendency as MDA levels. During normoxia, we found no significant difference in $\mathrm{LDH}$ levels between the gene silencing and normoxic control groups. In hypoxia, PHD1shRNA or Keap1shRNA treatment group led to a decrease in $\mathrm{LDH}$ release, furthermore, the $\mathrm{LDH}$ level of the co-transfected group was lower than that of the single groups.

\section{Effects of PHD1 and Keap1shRNAs on the Expression of Pro-fibrogenic Molecules in the Hypoxic AML12 Cells}

To clarify the function of PHD1 and Keap1 during fibrogenesis, the expression of some pro-fibrogenic molecules including 
COL1A1, transforming growth factor- $\beta 1$ (TGF- $\beta 1$ ), vascular endothelial growth factor-A (VEGF-A), and insulin-like growth factor 1 (IGF-1) was evaluated by qRT-PCR and western blot in the hypoxic AML12 cells. Upon exposure to hypoxia, mRNAs (Figure 5A) as well as proteins (Figures 5B,C) levels of COL1A1, TGF- $\beta 1$, VEGF, and IGF- 1 were upregulated, but the changes in TGF- $\beta 1$ and IGF-1 proteins were not statistically significant.

During hypoxia, single knockdown of PHD1 or Keap1 induced the downregulation of mRNAs (Figure 5A) and proteins (Figures 5B,C) levels of COL1A1, TGF- $\beta 1$, VEGF-A, and IGF1 compared to the control. More interestingly, the decrease was further enhanced when PHD1 and Keap1shRNAs were simultaneously introduced. To know whether the release of COL1A1 levels were affected by PHD1 and Keap1shRNAs, an ELISA assay was performed using the AML12 cells culture supernatants. The results showed that the levels of COL1A1 in the supernatants had the same trend of reduction as those in the cell lysates (Figure 5D).

\section{Effects of PHD1 and Keap1shRNAs-Treated Hypoxic AML12 Cells on the Expression of Fibrosis-Related Molecules in HSCs}

Considering the complex intrahepatic microenvironment, especially the complicated relationship between hepatocytes and HSCs, HSC-T6 cells were cultured in double-knockdown $\mathrm{CM}$ to better simulate the in vivo local environment, and then the corresponding fibrosis-related molecules were examined at the mRNAs and proteins levels. During hypoxia CM, COL1A1, $\alpha$-SMA, TGF- $\beta 1$, VEGF-A, and IGF-1 mRNAs (Figure 6A) and proteins (Figure 6B) in hypoxic single-knockdown CM were lower than those in the hypoxic control CM, and were further decreased in double-knockdown CM. However, no significant difference in these mRNAs and proteins expression was observed between the PHD1 or Keap1shNC CM and the control CM group.

\section{Effects of PHD1 and Keap1shRNAs-Treated Hypoxic AML12 Cells on Apoptosis and Proliferation of in HSCs}

Since double-knockdown CM deregulated the expression of fibrosis-related molecules in HSCs, we next detected how the apoptosis of HSCs was regulated using Annexin $\mathrm{V}$-APC/PI double staining. Apoptosis was increased in hypoxic single-knockdown CM, moreover, the apoptotic rate was further enhanced in both normoxic and hypoxic double-knockdown CM (Figures 7A-C). However, there was no significant difference between the knockdown
A
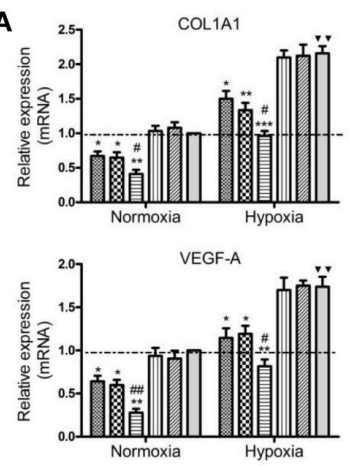

C
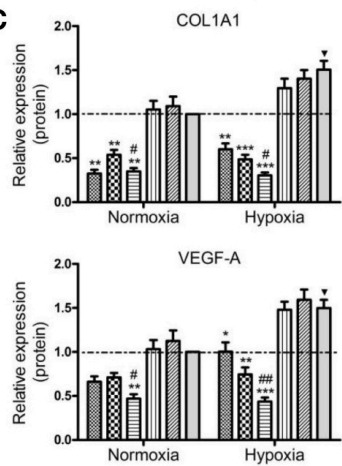

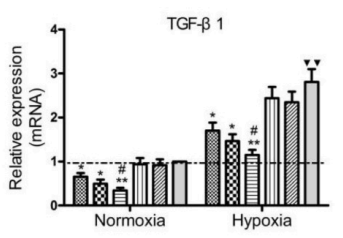

IGF-1
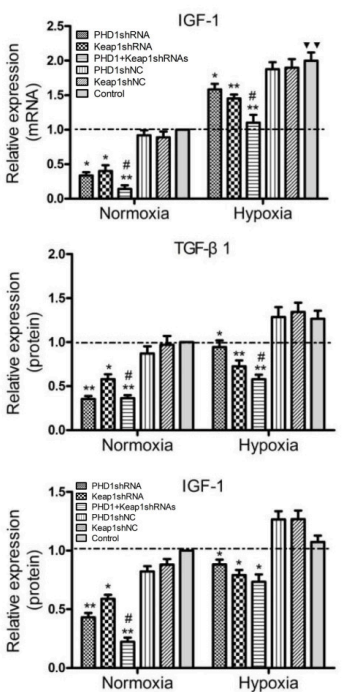

B

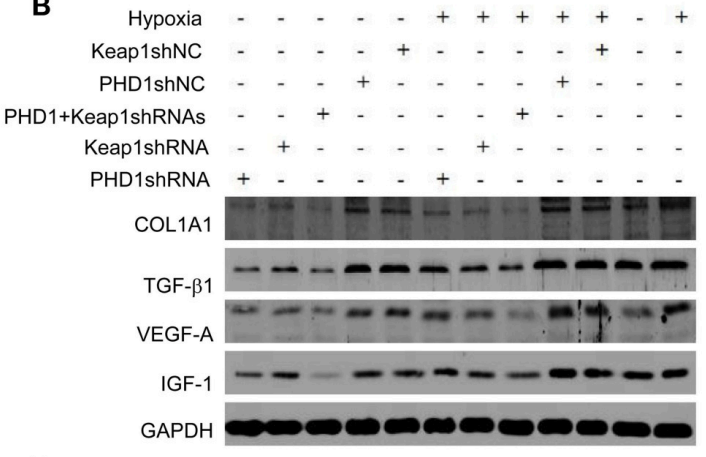

D

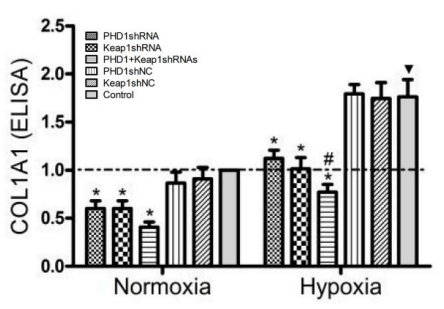

FIGURE 5 | Co-transfection of PHD1 and Keap1shRNAs inhibited expressions of pro-fibrogenic molecules during hypoxia. AML12 cells were co-transfected with PHD1 and Keap1 shRNAs, and then exposed to hypoxia for 12 h. (A) mRNAs and (B,C) proteins levels of COL1A1, TGF- $\beta 1$, VEGF-A, and IGF-1 were detected by qRT-PCR and western blot. (D) COL1A1 level was measured by ELISA. GAPDH was used as the internal standard, $n=3$. ${ }^{\star} p<0.05,{ }^{* *} p<0.01$, ${ }^{\star \star \star} p<0.001$ vs. respective normoxic and hypoxic control, $\# p<0.05, \# \# p<0.01$ vs. respective single-knockdown normoxic and hypoxic control, ${ }^{*} p<0.05$, $\boldsymbol{\nabla} \nabla_{p}<0.01$ vs. normoxic control. 
A

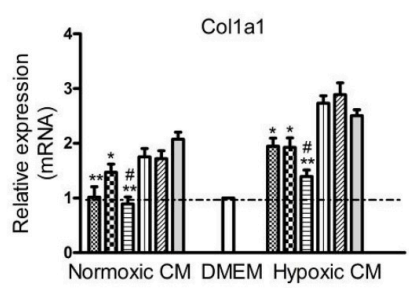

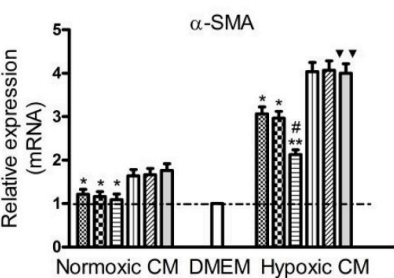
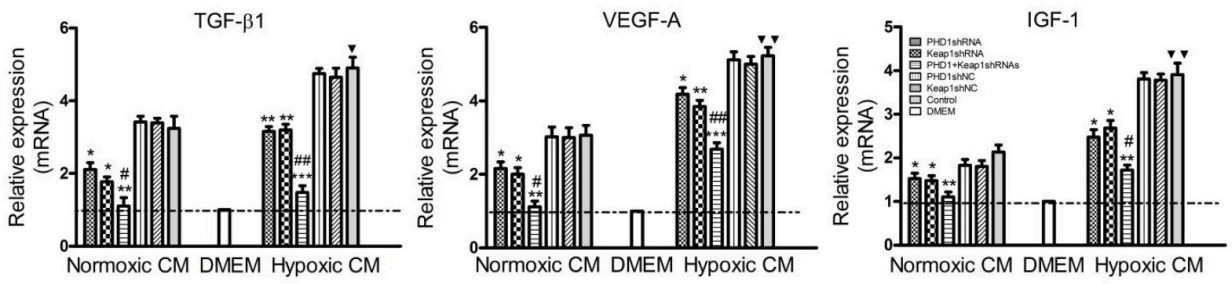

B

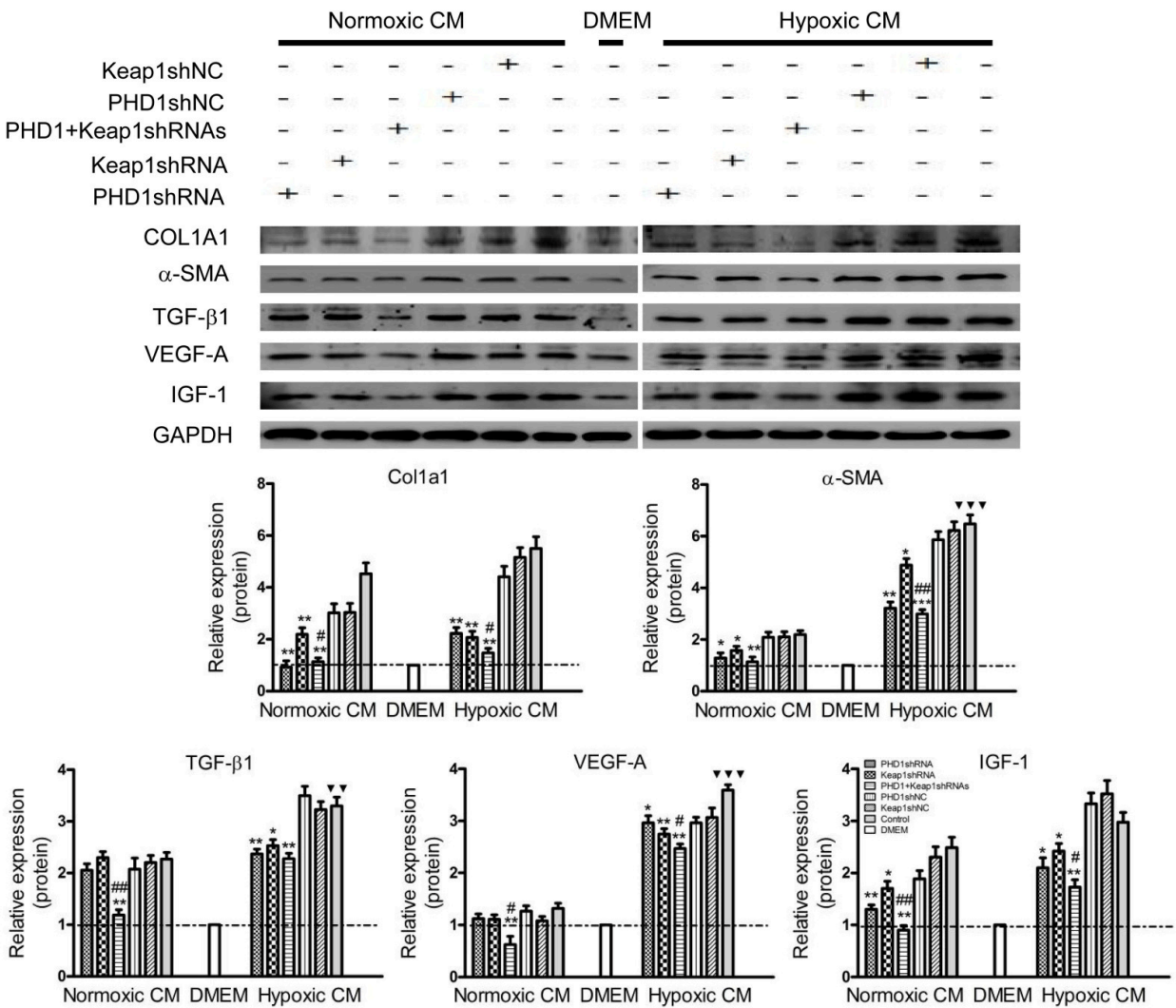

FIGURE 6 | Effects of PHD1 and Keap1shRNAs-treated hypoxic AML12 cells on the expression of fibrosis-related molecules. HSC-T6 cells were cultured in CM obtained from the supernatant of hypoxic PHD1 and/or Keap1 knockdown AML12 cells. (A) mRNAs and (B) proteins levels of COL1A1, $\alpha-S M A$, TGF- $\beta 1$, VEGF-A, and IGF-1 were measured via qRT-PCR and western blot. GAPDH was used as the internal standard, $n=3 .{ }^{*} p<0.05,{ }^{* *} p<0.01,{ }^{* \star *} p<0.001$ vs. respective normoxic and hypoxic control $\mathrm{CM}$, $\# p<0.05$, \#\# $p<0.01$ vs. respective normoxic and hypoxic single-knockdown $\mathrm{CM}$, ${ }^{\mathbf{\nabla}} p<0.05, \mathbf{\nabla} p<0.01$, $\boldsymbol{\nabla} \nabla \nabla_{p}<0.001$ vs. normoxic control CM.

$\mathrm{CM}$ groups and control CM group in normoxic CM. In addition, Figure 7D showed that cells cultured in CM enhanced cell viability compared with cells in DMEM, and the effect was enhanced in hypoxic CM. However, the cell proliferation of the HSC-T6 cells was reduced when cultured in single-transfected $\mathrm{CM}$ and was further weakened in the co-transfected CM.

\section{DISCUSSION}

In this study, we first successfully established a model of liver fibrosis induced by $\mathrm{CCl}_{4}$, which is a suitable model for exploring the underlying mechanisms involved in liver fibrosis (Brattin et al., 1985; Lee and Friedman, 2011). By analyzing the liver tissues from model animals with fibrosis, we observed that the 
A

PHD1shRNA Keap1shRNA PHD1+Keap1shRNAs PHD1shNC
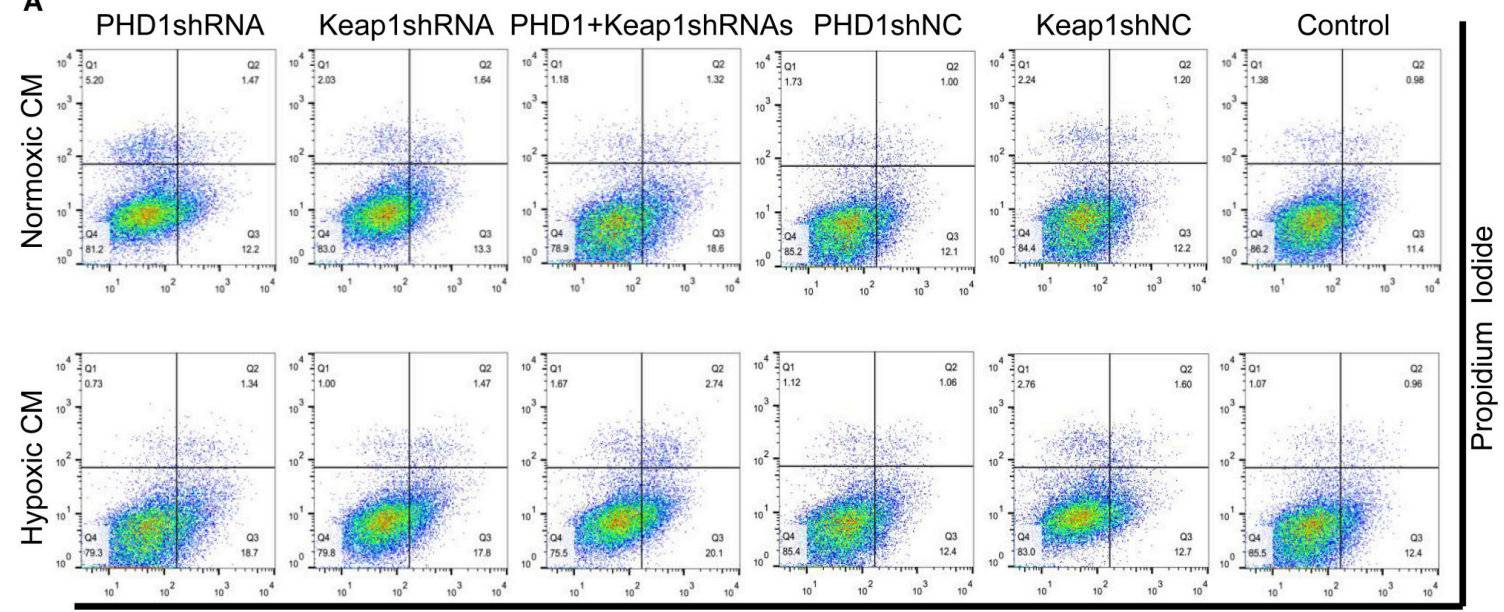

Annexin V APC

B

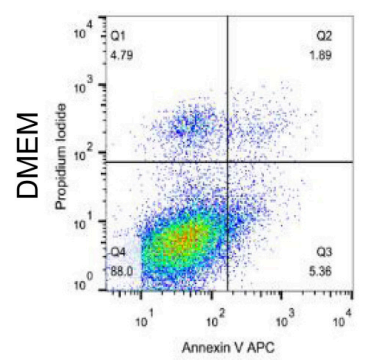

C

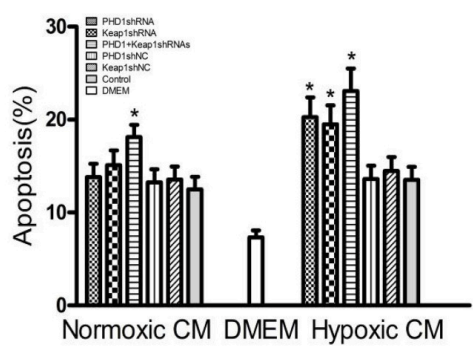

D

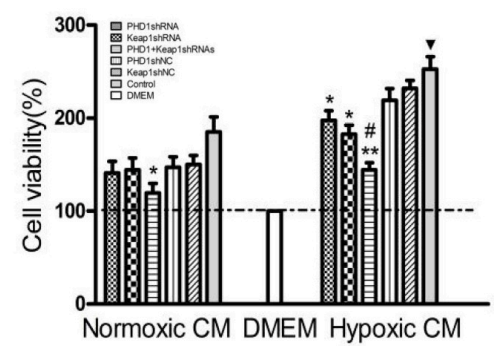

FIGURE 7 | Effects of PHD1 and Keap1shRNAs-treated hypoxic AML12 cells on the apoptosis and proliferation of HSCs. HSC-T6 cells were cultured in the CM obtained from the supernatant of hypoxic PHD1 and/or Keap1 AML12 cells. (A-C) AnnexinV-APC/PI double-stained cells undergoing apoptosis. (D) Cell viability was assessed by CCK-8 assay. Data are expressed as mean $\pm \mathrm{SEM}, n=3 .{ }^{*} p<0.05,{ }^{* *} p<0.01$ vs. respective normoxic and hypoxic control CM, $\#_{p}<0.05$ vs. respective normoxic and hypoxic single-knockdown $\mathrm{CM}, \mathbf{v}^{p}<0.05$ vs. normoxic control CM.

molecules related to hypoxia (HIF- $1 \alpha, \mathrm{HIF}-2 \alpha$ ) and oxidative stress (Keap1) were increased in hepatocytes, which provided a valid theoretical basis for the in vitro research.

Next, to elucidate the underlying mechanisms we chose hepatocytes as the primary target. To simulate the hypoxia in vitro, we used $1 \%$ oxygen (normoxia with $\sim 20 \%$ oxygen) in the incubators similar to Fingas' study (Fingas et al., 2011). In the hepatocytes, we observed that hypoxia only leads to a decrease in PHD1mRNA but not that of its protein expression, which implied that hypoxia controls the expression of PHD1 only at the transcript level. PHD1 is not only sensitive to oxygen concentration but also vulnerable to ROS generation (Kaelin and Ratcliffe, 2008). Our results also showed that knockdown of PHD1 increased the expression of both HIF- $2 \alpha$ mRNA and protein in the hepatocytes, but had no effect on HIF-1 $\alpha$ level. These findings were consistent with the previous studies reporting HIF- $2 \alpha$ as a major downstream mediator of PHD1 during hypoxia tolerance (Aragones et al., 2008; Schneider et al., 2010) and HIF-1 $\alpha$ may be regulated by the PHD2 pathway (Takeda et al., 2007). Furthermore, we found that Keap1shRNA could efficiently suppress Keap1 expression and enhance Nrf2 expression, which suggest that Keap1 negatively regulates Nrf2. Therefore, knockdown of Keap1 is vital for promoting Nrf2-mediated cytoprotection.

Until now, most anti-fibrotic approach researches are focused on either alleviating hypoxia or oxidative stress alone, however, a single treatment have showed an unsatisfactory result with a more side-effects (Halliwell, 2013; Kim and Yang, 2015; Biswas, 2016). Considering the interrelation between hypoxia and oxidative stress, in this experiment we used shRNAs to simultaneously knockdown the expressions of both PHD1 and Keap1 so that one sided dominance of either factor was eliminated. Our data suggest that optimal interference of PHD1 or Keap 1 was achieved at $12 \mathrm{~h}$ in hypoxic condition, therefore the co-transfected cells were cultured in $1 \%$ hypoxia up to this time point. We found that loss of PHD1 or Keap1 reduced oxidative stress by activating GSH and by subsequent inhibition of MDA. Schneider et al. reported that knockdown of PHD1 in the hepatocytes led to reduced oxidative stress (Schneider et al., 2010), and inhibition of Keap1 in the hepatocytes improved the resistance against oxidative stress by upregulating Nrf2 (Miyata et al., 2011), which are also inline with our findings and further supported our data. Importantly, our results indicated that 


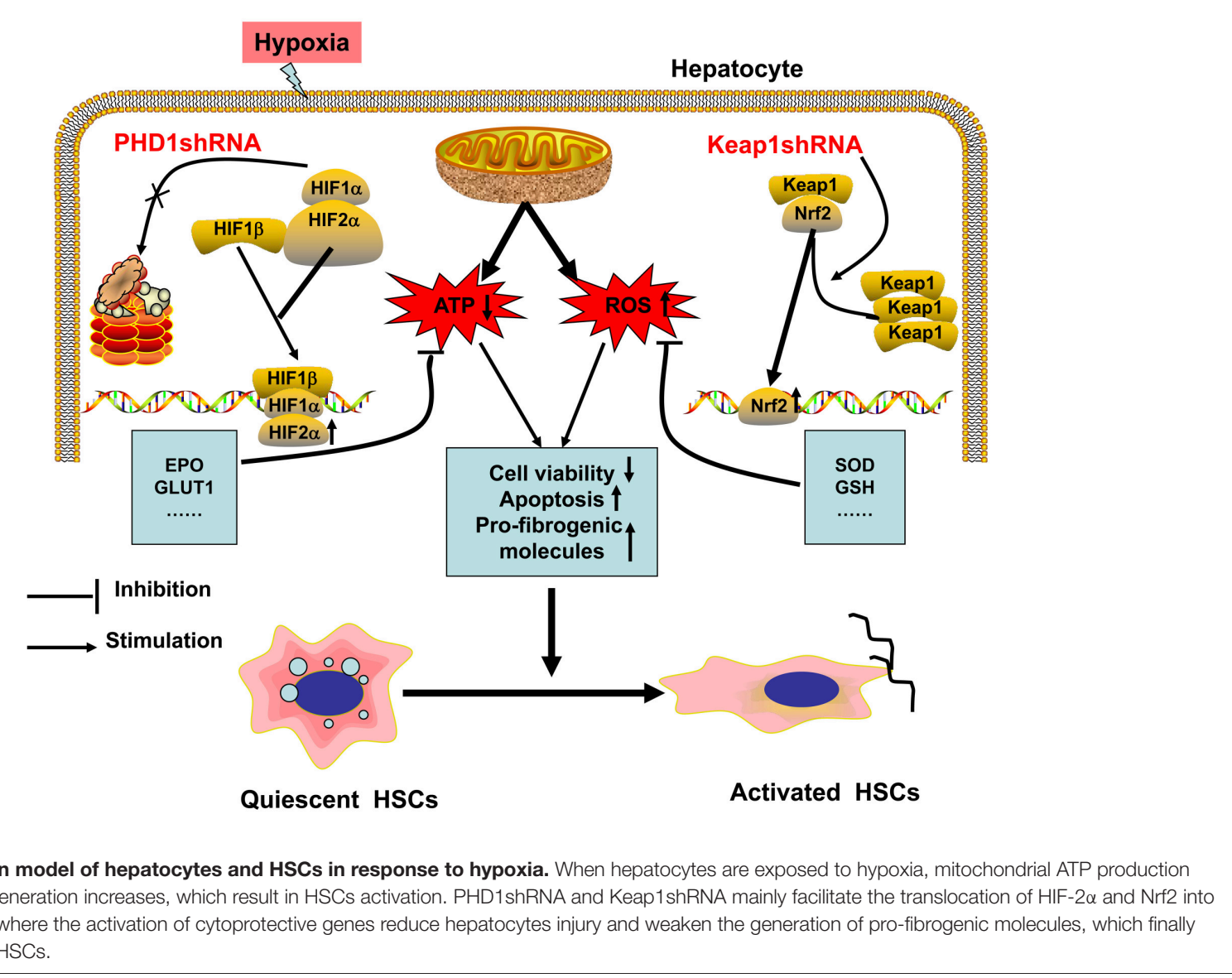

combined interference of PHD1 and Keap1 had a synergistic effect on the inhibition of oxidative stress.

Previous studies have shown that apoptosis was involved in the pathogenesis of hepatic fibrosis (Hamdy and El-Demerdash, 2012; Klein et al., 2012). In the study, we observed that apoptosis was decreased in the PHD1-knockdown hypoxic hepatocytes, and the anti-apoptotic effect observed was independent of the HIF signal pathway, which may be associated with the activation of NF-кB (Fitzpatrick et al., 2016). Meanwhile, Hamdy et al. reported that Nrf2 inhibited apoptosis via activation of the antiapoptotic Bcl-2 protein (Hamdy and El-Demerdash, 2012), and we also demonstrated that knockdown of Keap1 could inhibit the apoptosis of hepatocytes. Furthermore, combined knockdown of PHD1 and Keap1 led to stronger anti-apoptotic effects and improved viability of hepatocytes during hypoxia. Indeed, the hypoxia tolerance in the PHD1-knockdown hepatocytes is mainly as the result of the subsequent decrease in glucose oxidation and oxygen consumption.(Aragones et al., 2008; Schneider et al., 2010).

It is well known that cytokines play central roles in liver fibrosis by regulating inflammatary mediators toward various injuries (Marra, 2002). VEGF contributes to the process of liver fibrosis by promoting angiogenesis and activating HSCs (Yoshiji et al., 2003), TGF- $\beta 1$ plays a critical role in inducing the epithelial-mesenchymal transition of hepatocytes and thereby promoting collagen synthesis (Gressner and Weiskirchen, 2006; Kaimori et al., 2007), and IGF-1 also stimulates collagen synthesis and HSCs proliferation (Scharf et al., 1998). Ma et al. proposed that both HIF- $1 \alpha$ and HIF- $2 \alpha$ acted as positive regulators of VEGF (Ma et al., 2014), moreover the changes in TGF- $\beta 1$ expression was in HIF dependent manner ( $\mathrm{Qu}$ et al., 2015). However, our data indicated that the levels of the pro-fibrogenic molecules-COL1A1, TGF- $\beta 1$, VEGF$A$, and IGF-1 were downregulated in PHD1 knockdown cells during hypoxia, and this may be because TGF- $\beta 1$ and VEGF-A may also be under regulation of ROS besides HIF (Kuroki et al., 1996; Jobling et al., 2006). In addition, we found that the knockdown of Keap1 could inhibit the expression of pro-fibrogenic molecules. Furthermore, concomitant knockdown of both PHD1 and Keap1 synergistically reduced the expression of pro-fibrogenic molecules, thereby inhibiting the progression of fibrosis, however, the specific mechanism is still unclear.

Hypoxic hepatocytes are capable of promoting the expression of TGF- $\beta 1$ and VEGF-A, which further activates HSCs (Qu et al., 2015). In addition, hepatocytes undergoing apoptosis and oxidative stress which in turn activates HSCs (Zhan et al., 2006; Subhadip et al., 2011), Therefore, considering the complex intrahepatic microenvironment and the interaction between hepatocytes and HSCs (Ayako et al., 2004), we used CM from the 
supernatant of hypoxic hepatocytes to culture HSCs. Our data showed that double-knockdown CM from hepatocytes could suppress the HSCs activation and prevent the progression of fibrosis. Alternatively, prompting apoptosis of activated HSCs may also be an alternative approach to resolve fibrosis (Iredale, 2001). It is worthly noted that double-knockdown CM could induce an increased apoptosis of HSCs, thereby inhibiting their proliferation.

The possible mechanism of hypoxia-induced hepatocytes injury are illustrated in Figure 8. Hypoxia is the initiator of this process, and can induce oxidative stress, which decreases the cellular acitivity, enhances apoptosis, promotes expression of pro-fibrogenic molecules, and hence activates HSC. In our study, we have demonstrated that single-knockdown of PHD1 or Keap1 could alleviate hepatocellular hypoxic injury by upregulating the expression of HIF- $2 \alpha$ and Nrf2 respectively, which then inhibits the HSC activation (Figure 8), moreover, double-knockdown of PHD1 and Keap1 could synergistically enhance this effect. Collectively, these findings will provide new

\section{REFERENCES}

Aragones, J., Schneider, M., Van Geyte, K., Fraisl, P., Dresselaers, T., Mazzone, M., et al. (2008). Deficiency or inhibition of oxygen sensor Phd1 induces hypoxia tolerance by reprogramming basal metabolism. Nat. Genet. 40, 170-180. doi: 10.1038/ng.2007.62

Ayako, M., Ian, M., Philip, W. S., Paul, A. H., Beth, L. M., Michael, N. T. et al. (2004). Role of hepatic stellate cell/hepatocyte interaction and activation of hepatic stellate cells in the early phase of liver regeneration in the rat. J. Hepatol. 40, 910-916. doi: 10.1016/j.jhep.2004.02.005

Begriche, K., Massart, J., Robin, M. A., Borgne-Sanchez, A., and Fromenty, B. (2011). Drug-induced toxicity on mitochondria and lipid metabolism: mechanistic diversity and deleterious consequences for the liver. J. Hepatol. 54, 773-794. doi: 10.1016/j.jhep.2010.11.006

Biswas, S. K. (2016). Does the interdependence between oxidative stress and inflmm ation explain the antioxidant paradox? Oxid. Med. Cell Longev. 2016:5698931. doi: 10.1155/2016/5698931

Bogdanos, D. P., Gao, B., and Gershwin, M. E. (2013). Liver immunology. Compr. Physiol. 3, 567-598. doi: 10.1002/cphy.c120011

Brattin, W. J., Glende, E. A. Jr., and Recknagel, R. O. (1985). Pathological mechanisms in carbon tetrachloride hepatotoxicity. J. Free Radic. Biol. Med. 1, 27-38. doi: 10.1016/0748-5514(85)90026-1

Cannito, S., Paternostro, C., Busletta, C., Bocca, C., Colombatto, S., Miglietta, A., et al. (2014). Hypoxia, hypoxia-inducible factors and fibrogenesis in chronic liver diseases. Histol. Histopathol. 29, 33-44. doi: 10.14670/HH-29.33

Chen, S., Zou, L., Li, L., and Wu, T. (2013). The protective effect of glycyrrhetinic acid on carbon tetrachloride-induced chronic liver fibrosis in mice via upregulation of Nrf2. PLoS ONE 8:e53662. doi: 10.1371/journal.pone. 0053662

Fingas, C. D., Bronk, S. F., Werneburg, N. W., Mott, J. L., Guicciardi, M. E., Cazanave, S. C., et al. (2011). Myofibroblast-derived PDGF-BB promotes Hedgehog survival signaling in cholangiocarcinoma cells. Hepatology 54, 2076-2088. doi: 10.1002/hep.24588

Fitzpatrick, S. F., Fabian, Z., Schaible, B., Lenihan, C. R., Schwarzl, T., Rodriguez, J., et al. (2016). Prolyl hydroxylase-1 regulates hepatocyte apoptosis in an NF-кB -dependent manner. Biochem. Biophys. Res. Commun. 474, 579-586. doi: 10.1016/j.bbrc.2016.04.085

Ghatak, S., Biswas, A., Dhali, G. K., Chowdhury, A., Boyer, J. L., and Santra, A. (2011). Oxidative stress and hepatic stellate cell activation are key events in arsenic induced liver fibrosis in mice. Toxicol. Appl. Pharmacol. 251, 59-69. doi: 10.1016/j.taap.2010.11.016 targets for developing new effective clinical drugs to treat liver fibrosis. However, further studies are needed to confirm our hypothesis in vivo.

\section{AUTHOR CONTRIBUTIONS}

Conceived and designed the experiments: JL, YL, and PC; Performed the experiments: JL, LL, ZW, CS, ZC, XZ, and FD; Analyzed the data and wrote the paper: JL and YL; Revised the paper: YL and PC.

\section{FUNDING}

This work was financially supported by the National Nature Science Foundation of China (No 81370868), the Fundamental Research Funds for the Central Universities and the Scientific Research Innovation Program for Graduate Students of Jiangsu Province, China (No KYLX16_0298), and (No KYZZ15_0062).

Gressner, A. M., and Weiskirchen, R. (2006). Modern pathogenetic concepts of liver fibrosis suggest stellate cells and TGF- $\beta$ as major players and therapeutic targets. J. Cell. Mol. Med. 10, 76-99. doi: 10.1111/j.1582-4934.2006.tb00292.x

Halliwell, B. (2013). The antioxidant paradox: less paradoxical now? Br. J. Clin. Pharmacol. 75, 637-644. doi: 10.1111/j.1365-2125.2012.04272.x

Hamdy, N., and El-Demerdash, E. (2012). New therapeutic aspect for carvedilol: antifibrotic effects of carvedilol in chronic carbon tetrachloride-induced liver damage. Toxicol. Appl. Pharmacol. 261, 292-299. doi: 10.1016/j.taap. 2012.04.012

Inoue, A., Sawata, S. Y., and Taira, K. (2006). Molecular design and delivery of siRNA. J. Drug. Target. 14, 448-455. doi: 10.1080/10611860600845397

Iredale, J. P. (2001). Hepatic stellate cell behavior during resolution of liver injury. Semin. Liver Dis. 21, 427-436. doi: 10.1055/s-2001-17557

Jobling, M. F., Mott, J. D., Finnegan, M. T., Jurukovski, V., Erickson, A. C., Walian, P. J., et al. (2006). Isoform-specific activation of latent transforming growth factor $\beta$ (LTGF- $\beta$ ) by reactive oxygen species. Radiat. Res. 166, 839-848. doi: $10.1667 /$ RR0695.1

Kaelin, W. G. Jr., and Ratcliffe, P. J. (2008). Oxygen sensing by metazoans: the central role of the HIF hydroxylase pathway. Mol. Cell. 30, 393-402. doi: 10.1016/j.molcel.2008.04.009

Kaimori, A., Potter, J., Kaimori, J. Y., Wang, C., Mezey, E., and Koteish, A. (2007). Transforming growth factor- $\beta 1$ induces an epithelial-to-mesenchymal transition state in mouse hepatocytes in vitro. J. Biol. Chem. 282, 22089-22101. doi: 10.1074/jbc.M700998200

Kamura, T., Sato, S., Iwai, K., Czyzyk-Krzeska, M., Conaway, R. C., and Conaway, J. W. (2000). Activation of HIF1 $\alpha$ ubiquitination by a reconstituted von HippelLindau (VHL) tumor suppressor complex. Proc. Natl. Acad. Sci. U.S.A. 97, 10430-10435. doi: 10.1073/pnas.190332597

Kang, M. I., Kobayashi, A., Wakabayashi, N., Kim, S. G., and Yamamoto, M. (2004). Scaffolding of Keap1 to the actin cytoskeleton controls the function of Nrf2 as key regulator of cytoprotective phase 2 genes. Proc. Natl. Acad. Sci. U.S.A. 101, 2046-2051. doi: 10.1073/pnas.0308347100

Kim, S. Y. and Yang, E. G. (2015). Recent advances in developing inhibitors for hypoxiainducible factor prolyl hydroxylases and their therapeutic implications. Molecules 19, 20551-20568. doi: 10.3390/molecules201119717

Klein, S., Klosel, J., Schierwagen, R., Korner, C., Granzow, M., Huss, S., et al. (2012). Atorvastatin inhibits proliferation and apoptosis, but induces senescence in hepatic myofibroblasts and thereby attenuates hepatic fibrosis in rats. Lab. Invest. 92, 1440-1450. doi: 10.1038/labinvest.2012.106

Kuroki, M., Voest, E. E., Amano, S., Beerepoot, L. V., Takashima, S., Tolentino, M., et al. (1996). Reactive oxygen intermediates increase vascular endothelial 
growth factor expression in vitro and in vivo. J. Clin. Invest. 98, 1667-1675. doi: 10.1172/JCI118962

Lee, U. E., and Friedman, S. L. (2011). Mechanisms of hepatic fibrogenesis. Best. Pract. Res. Clin. Gastroenterol. 25, 195-206. doi: 10.1016/j.bpg.2011.02.005

Ma, L., Li, G., Zhu, H., Dong, X., Zhao, D., Jiang, X., et al. (2014). 2-Methoxyestradiol synergizes with sorafenib to suppress hepatocellular carcinoma by simultaneously dysregulating hypoxia-inducible factor-1 and -2 . Cancer Lett. 355, 96-105. doi: 10.1016/j.canlet.2014.09.011

Marra, F. (2002). Chemokines in liver inflammation and fibrosis. Front. Biosci. 7, d1899-d1914. doi: 10.2741/a887

Minamishima, Y. A., Moslehi, J., Padera, R. F., Bronson, R. T., Liao, R., and Kaelin, W. G. Jr. (2009). A feedback loop involving the Phd3 prolyl hydroxylase tunes the mammalian hypoxic response in vivo. Mol. Cell. Biol. 29, 5729-5741. doi: 10.1128/MCB.00331-09

Miyata, T., Takizawa, S., and Van Ypersele De Strihou, C. (2011). Hypoxia. 1. Intracellular sensors for oxygen and oxidative stress: novel therapeutic targets. Am. J. Physiol. Cell. Physiol. 300, C226-C231. doi: 10.1152/ajpcell.00430.2010

Mormone, E., Lu, Y., Ge, X., Fiel, M. I., and Nieto, N. (2012). Fibromodulin, an oxidative stress-sensitive proteoglycan, regulates the fibrogenic response to liver injury in mice. Gastroenterology 142, 612-621.e5. doi: 10.1053/j.gastro.2011.11.029

Nakanishi, K., Tajima, F., Nakamura, A., Yagura, S., Ookawara, T., Yamashita, H., etal. (1995). Effects of hypobaric hypoxia on antioxidant enzymes in rats. J. Physiol. 489 (Pt 3), 869-876. doi: 10.1113/jphysiol.1995.sp021099

Nieto, N., Friedman, S. L., and Cederbaum, A. I. (2002). Cytochrome P450 2E1derived reactive oxygen species mediate paracrine stimulation of collagen I protein synthesis by hepatic stellate cells. J. Biol. Chem. 277, 9853-9864. doi: 10.1074/jbc.M110506200

Nimker, C., Kaur, G., Revo, A., Chaudhary, P., and Bansal, A. (2015). Ethyl 3,4dihydroxy benzoate, a unique preconditioning agent for alleviating hypoxiamediated oxidative damage in L6 myoblasts cells. J. Physiol. Sci. 65, 77-87. doi: 10.1007/s12576-014-0348-1

Okada, K., Shoda, J., Taguchi, K., Maher, J. M., Ishizaki, K., Inoue, Y., et al. (2009). Nrf2 counteracts cholestatic liver injury via stimulation of hepatic defense systems. Biochem. Biophys. Res. Commun. 389, 431-436. doi: 10.1016/j.bbrc.2009.08.156

Qu, K., Yan, Z., Wu, Y., Chen, Y., Qu, P., Xu, X., et al. (2015). Transarterial chemoembolization aggravated peritumoral fibrosis via hypoxia-inducible factor-1 $\alpha$ dependent pathway in hepatocellular carcinoma. J. Gastroenterol. Hepatol. 30, 925-932. doi: 10.1111/jgh.12873

Rankin, E. B., Rha, J., Selak, M. A., Unger, T. L., Keith, B., Liu, Q., et al. (2009). Hypoxia-inducible factor 2 regulates hepatic lipid metabolism. Mol. Cell. Biol. 29, 4527-4538. doi: 10.1128/MCB.00200-09
Scharf, J. G., Knittel, T., Dombrowski, F., Muller, L., Saile, B., Braulke, T., et al. (1998). Characterization of the IGF axis components in isolated rat hepatic stellate cells. Hepatology 27, 1275-1284. doi: 10.1002/hep.510 270513

Schneider, M., Van Geyte, K., Fraisl, P., Kiss, J., Aragones, J., Mazzone, M., et al. (2010). Loss or silencing of the PHD1 prolyl hydroxylase protects livers of mice against ischemia/reperfusion injury. Gastroenterology 138, 1143-1154.e1-2. doi: 10.1053/j.gastro.2009.09.057

Subhadip, G., Ayan, B., Gopal, K. D., Abhijit, C., James, L. B., and Amal, S. (2011). Oxidative stress and hepatic stellate cell activation are key events in arsenic induced liver fibrosis in mice. Toxicol. Appl. Pharm. 251, 59-69 doi: 10.1016/j.taap.2010.11.016

Takeda, K., Cowan, A., and Fong, G. H. (2007). Essential role for prolyl hydroxylase domain protein 2 in oxygen homeostasis of the adult vascular system. Circulation 116, 774-781. doi: 10.1161/CIRCULATIONAHA.107. 701516

Tanaka, Y., Ikeda, T., Yamamoto, K., Ogawa, H., and Kamisako, T. (2012). Dysregulated expression of fatty acid oxidation enzymes and iron-regulatory genes in livers of Nrf2-null mice. J. Gastroenterol. Hepatol. 27, 1711-1717. doi: 10.1111/j.1440-1746.2012.07180.x

Yang, J. J., Tao, H., Huang, C., and Li, J. (2013). Nuclear erythroid 2-related factor 2: a novel potential therapeutic target for liver fibrosis. Food. Chem. Toxicol. 59, 421-427. doi: 10.1016/j.fct.2013.06.018

Yoshiji, H., Kuriyama, S., Yoshii, J., Ikenaka, Y., Noguchi, R., Hicklin, D. J., et al. (2003). Vascular endothelial growth factor and receptor interaction is a prerequisite for murine hepatic fibrogenesis. Gut 52, 1347-1354. doi: $10.1136 /$ gut.52.9.1347

Zhan, S. S., Jiang, J. X., Wu, J., Halsted, C., Friedman, S. L., Zern, M. A., et al. (2006). Phagocytosis of apoptotic bodies by hepatic stellate cells induces NADPH oxidase and is associated with liver fibrosis in vivo. Hepatology 43, 435-443. doi: 10.1002/hep. 21093

Conflict of Interest Statement: The authors declare that the research was conducted in the absence of any commercial or financial relationships that could be construed as a potential conflict of interest.

Copyright (c) $2017 \mathrm{Liu}, \mathrm{Li}$, Liu, Wang, Shi, Cheng, Zhang, Ding and Chen. This is an open-access article distributed under the terms of the Creative Commons Attribution License (CC BY). The use, distribution or reproduction in other forums is permitted, provided the original author(s) or licensor are credited and that the original publication in this journal is cited, in accordance with accepted academic practice. No use, distribution or reproduction is permitted which does not comply with these terms. 